\title{
Self-learning of the direct soil tomography problem using a specific educational software
}

\section{Sánchez Pérez, Juan Francisco ${ }^{a}$; Alhama Manteca, Iván ${ }^{\text {b }}$ García Ros, Gonzalob and Cánovas Vidal, Manuel. ${ }^{\mathrm{c}}$}

${ }^{a}$ Departmento de Física Aplicada, Universidad Politécnica de Cartagena, Spain. ${ }^{\mathrm{b}}$ Departmento de Ingeniería Civil, Universidad Politécnica de Cartagena, Spain. ${ }^{\mathrm{c}}$ Departmento de Ingeniería Metalúrgica y Minera, Universidad Católica del Norte, Chile.

\begin{abstract}
The educational tool DITPRO has been created to simulate problems of direct soil tomography problem. The introduction of data is very easy for the students, because the software has an interactive grid window. Moreover, provides to the users an easy handling and powerful calculations. Its graphical environment allows access to a graphical representation of potential functions. The software presents, like commercial softwares, the most common options (e.g., save and open cases files and representations, etc.). Although the program is created to be used in several fields, among others as investigation tool, the mainly use of interest for this work is as educational tool. Another important use is as low-cost laboratory practices. The student has the possiblity to obtain system information of the Spice code text file, whose rules are well known by students with knowledge of electrical circuit theory. The student must to design and implement several sceneries and explain it to check his/her understanding of the problem.
\end{abstract}

Keywords: educational software; teaching in engineering; design mathematical models; Spice code; Soil tomography. 


\section{Introduction}

Faced with the possibility of teaching in a course of Engineering Master, the main question that appears is what would be the best best tool for students to understand the different water and soil engineering problems. Given the great experience of the research group components in numerical simulation using the network method and Spice code, the idea of developing different softwares with a friendly environment to allow students the understanding of these difficult problems arose. Although the software can be used by any student learning soil engineering, the main careers to which is focused are civil and building engineering.

The network method is firstly based on the electrical analogy and it is detailed in several engineering books. On the other hand, it is used as an educational tool to explain the relationship between mathematical models and physical and engineering processes [Sanchez-Perez et al. (2016a)]. This procedure, analogy between equations and physical problems, is an unquestionable educational subject since many engineering or physical models use the same partial or differential differential equations. [Mills (1999) and Chapman (1960) and González-Fernández C.F. and Horno J. (2002)]. In previous works have been presented the use successfully of educational softwares in classroom experiences [Sanchez-Perez and Alhama (2016)].

In this work is presented a software capable of simulating 2-D, direct tomography problem: DITPRO [Sanchez-Perez et al. (2016b)] and its use as educational tool. The software was created by the research group "Network Simulation" of the Universidad Politécnica de Cartagena.

Although DITPRO is created to be used in several fields, among others as investigation tool, the mainly use of interest for this work is as educational tool, allowing to explain the relation between physical processes and mathematical equations of the model. Another important use is as low-cost laboratory practices.

Finally, DITPRO, which makes use of analogy or equivalence between electric transport and transport of current in soil is presented to the user through an environment of pleasant communication, type windows, which leads to actions "step by step" and possible options such as selection and problem definition, data entry, file creation and manipulation of models, simulation options, advanced simulation, presentation of results, etc. Files network models developed by DITPRO run in Spice softwares and the simulation results are provided directly in the graphical environment or through appropriate manipulations, in the graphical environment of the program itself. 


\section{Theoretical basis of direct problems in tomography}

The electrical tomography is a tool whose purpose is to determine the resistivity for the characterization of the subsoil. The process consists in introducing a continuous electric current into the ground through a pair of electrodes (current) that are located in determined positions, measuring the difference of potential (d.d.p.) in specific locations, by another pair of electrodes located on the surface of the terrain. This d.d.p. is caused by the input current. From the value of the applied current and the measured voltage, the application of the law of Ohm allows to determine the apparent resistivity of the subsurface, characteristic parameter of each type of material.

In tomography, the direct problem is to find the distribution of electric potentials and currents throughout the domain, particularly on the surface of the terrain, from the properties and geometry of the medium and the boundary conditions, a potential difference between two points. From the set of aligned points where the surface electrodes are placed, generally symmetrical with respect to the vertical that separates the domain into two equal parts.

For this boundary condition, the cells in which the potentials are applied must be sufficiently far from the side and bottom boundaries for this condition to be satisfied; Thus, $\mathrm{L}_{\mathrm{a}}$ is the distance between the point of application of voltage $\mathrm{V}(+1$ or -1$)$ and $\mathrm{L}_{\mathrm{b}}$ is half the horizontal length of the domain, Figure 1.

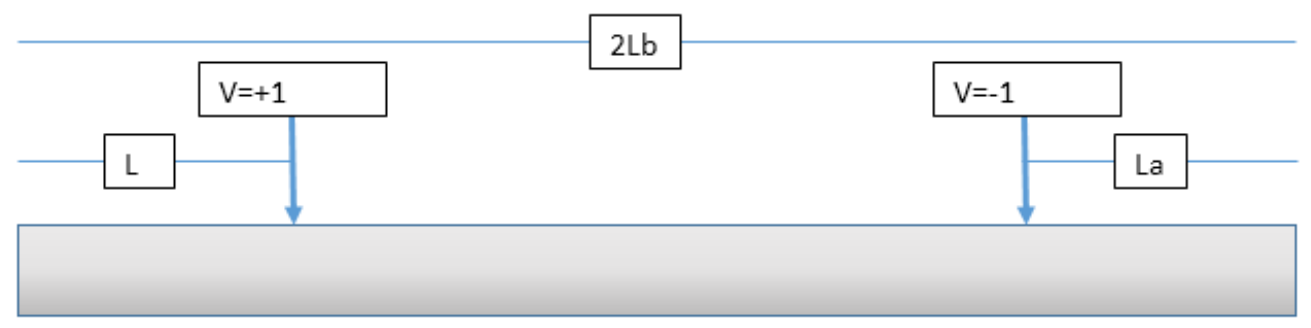

Figure 1. Measurement diagram of the tomograph

The solution of the direct problem and the model allows the visualization of both current and equipotential voltage lines and the analisys the patterns distortions of these lines associated with the presence of layers, subregions, holes ... of different electrical conductivities. Notice that the problem is reduced to the study of the electric fields that are created in the half-space of an electric dipole, that of the ground. The other half space is assumed to be null conductivity.

On the other hand, the 3-D effect is neglected in this software, this is equivalent to assuming that our results would be valid for 2 -D problems, that is, the potentials $(+1$ and 1) would apply to two infinite lines perpendicular to the surface of the domain and over the 
surface of the terrain. The potentials of the centers of each cell are read directly in the output file whereas the electric currents (which can also be read from the output file since they are the currents in the resistances of the cells) are obtained more directly by drawing the gradients of the equipotential curves.

\section{DITPRO}

Figure 2 shows a simplified scheme of the basic operation DITPRO. Its implementation gives access directly to the input data: geometry crosslinking, physical characteristics, boundary conditions, etc. Once completed the specification of the problem can create a basic text file model that allows direct manipulation and modification. Because of all this, DITPRO provides to the users an easy handling and thanks to spice code, powerful calculations.



Figure 2. Simplified flow diagram of the program. 
The introduction of data is very easy for the students, at the start of the DITPRO software, the main window is opened, where the information necessary to perform a simulation by direct problem analysis in soil electrical tomography is entered. The basic data to be supplied to the program are: Number of diodes, Separation between diodes, Depth of terrain to be studied, Parameters Geometric (width of vertical regions and amount and thickness of layers or extracts within the terrain) together with a mesh factor fa, fb, fc, f1, f2 and f3) to each of these, and the value of the physical parameters of each region. All the data are introduced as appropriate screens such as those shown in Figure 3. All this information and more is detailed in the "Help" windows.

After this data has been entered, the type and quality of the required result (Maximum Intensity, Surface Potential and potential in the field in low or high quality) is selected and the software is executed. Automatically the program will perform multiple simulations by varying the position of the active diodes from the central diodes and with each simulation opening towards the next until reaching the extreme diodes or maximum opening.

Once the model has been numerically solved in the Spice code, the screen "Simulation results" is shown. The graphical environment DITPRO allows access to a graphical representation of maximum intensity, surface potential and land potential. Access to these representations is straightforward once simulated the model.

It stores the results obtained from the simulations in an ".xls" format file in which the first column of the results represents half the distance between the active diodes $(d / 2)$ and the second column is the measured value. On the other hand, DITPRO incorporates a graphics presentation using Matlab, Figure 4. Moreover presents, like commercial software's, the most common options (e.g., save and open cases files, save animations and representations, etc.). 


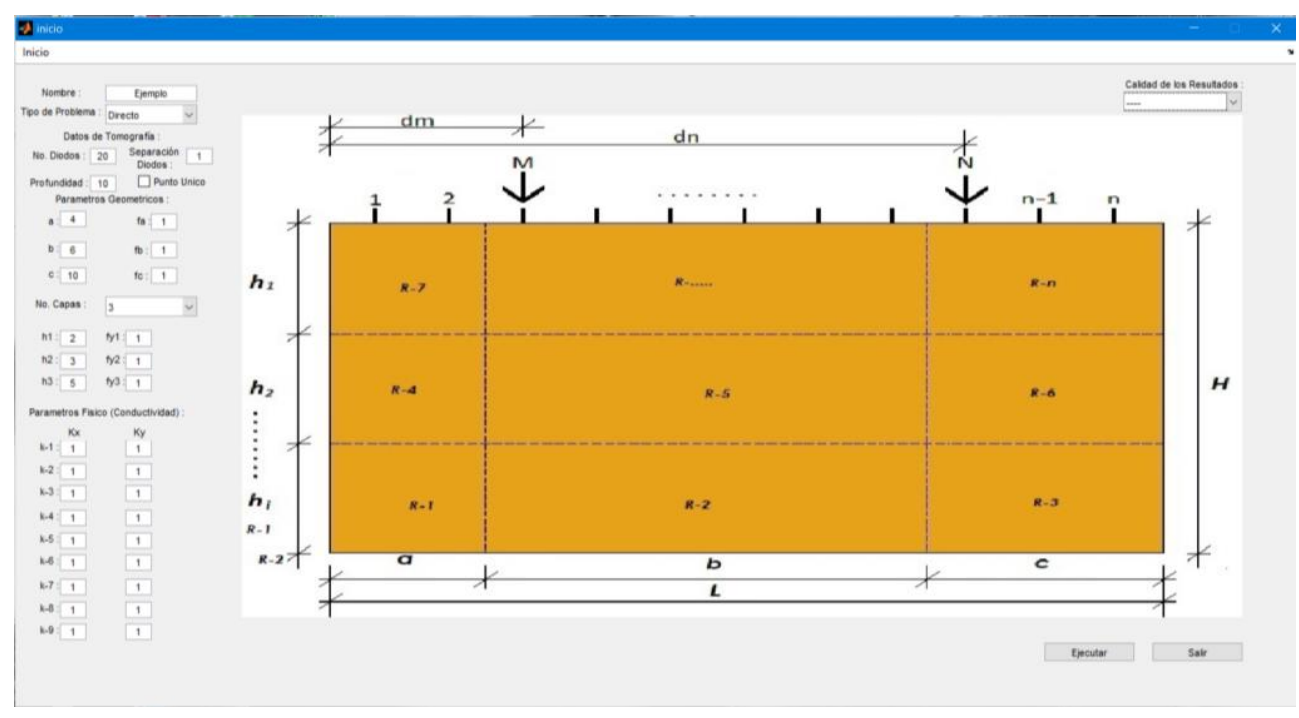

Figure 3. Editing geometrical and physical parameters of the problem.

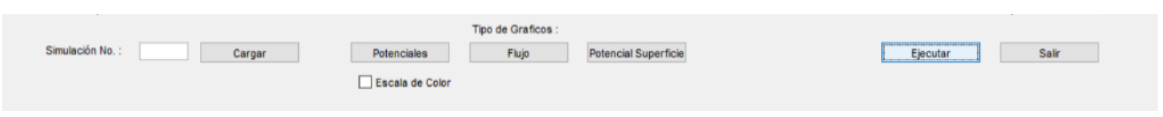

Figure 4. Results graph options

The dependability and efficiency of the Spice code has been probed by a great number of works in different fields of engineering, such as heat transfer, chemical, elasticity and so on, already published in the scientific literature.

\section{Proposal for useroom in class: direct problems in tomography}

Master course of CIETAT devoted to water and soil engineering has been teaching since 2012 in the Universidad Politécnica de Cartagena. In the part of the terrain, the students are introduced in the equations that characterize it. From the network simulation group, we discussed how to facilitate the learning of these systems. The result is DITPRO software.

For the proposal of use in classroom, the student can propose and solve many scenarios. The student must determine the real parameters (physical characteristics, initial conditions, location of the connection points of the electrodes, etc.) that are required to define the tomographic problem and access the simulation as if it were a field work.

The main pararmeters to introduce are the electrical capacity of the subsoil (or its layers), property that has the bodies associated with the retention of electric charge in its inside, electrical conductivity, the ability of the ground to allow the passage of electric charges through it, point of application of the excitation electrodes, are the points in the field where 
the constant current power source will be applied and time, it is time of application of the load, necessary to reach a steady state. Figure 3 shows a land rectangular domain, points of application of voltage and all parameters to introduce.

Once the problem is solved by the software (Figures 5 to 8), the student must interpret the results and their association with the govern equation. Finally, each student must explain the meaning of the solutions obtained with the software and the physical meaning of it.

The evaluation of student's learning is done by posing a real problem that must be solved explaining each of the solution characteristics obtained and its application to the problem optimization.

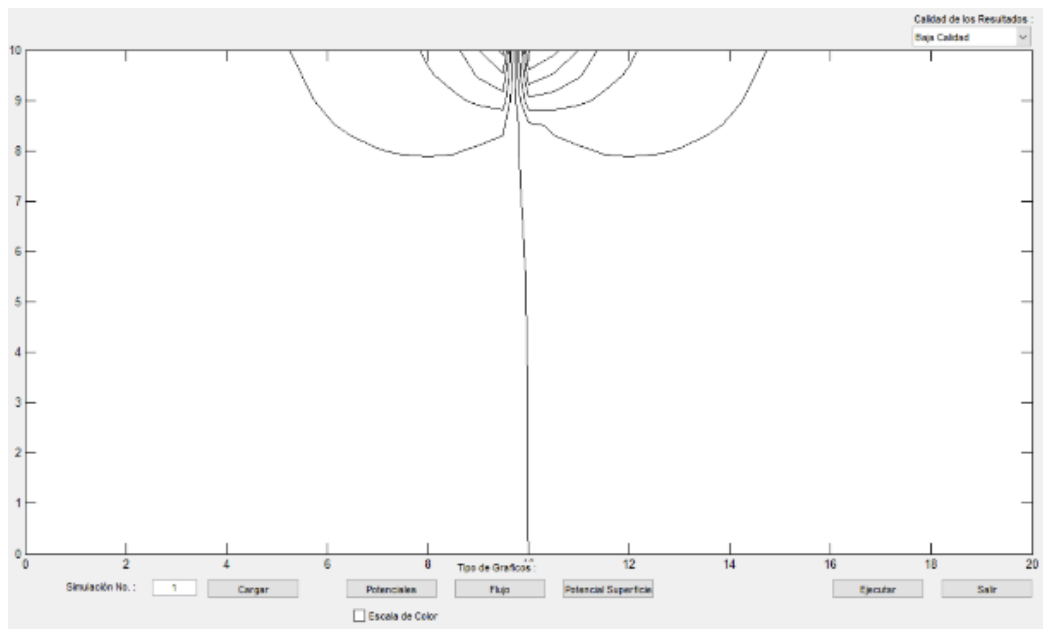

Figure 5. Potential in Land

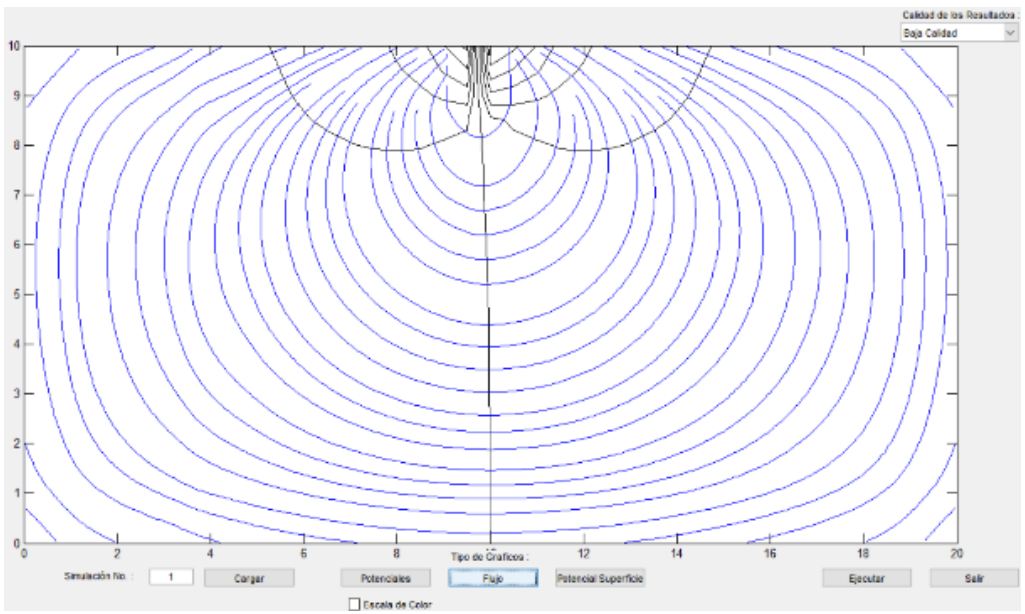

Figure 6. Potential and Flow 


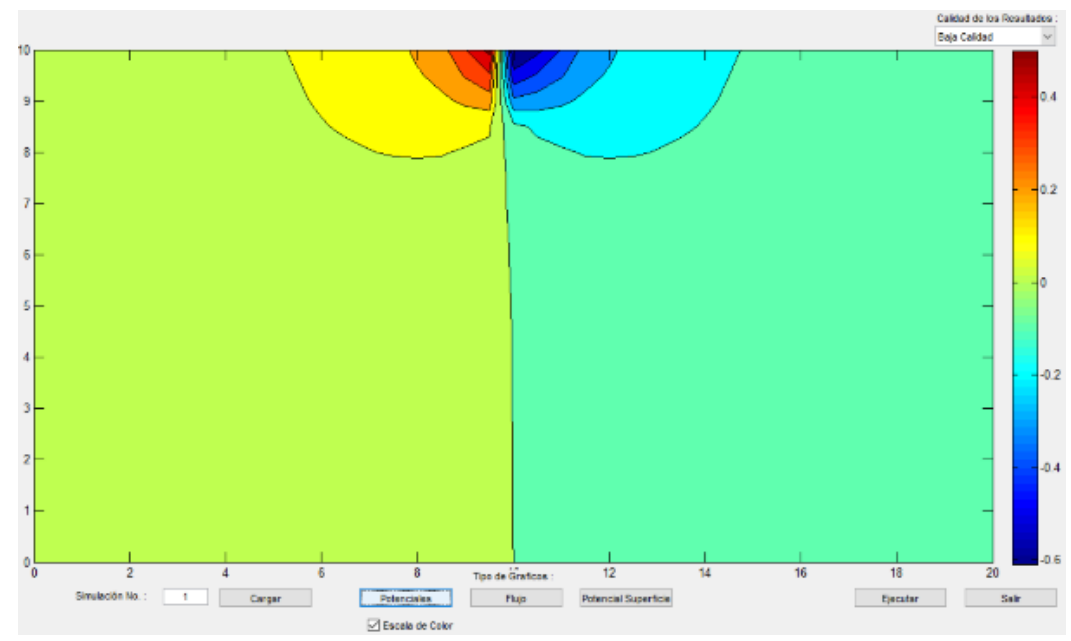

Figure 7. Potential with color scale

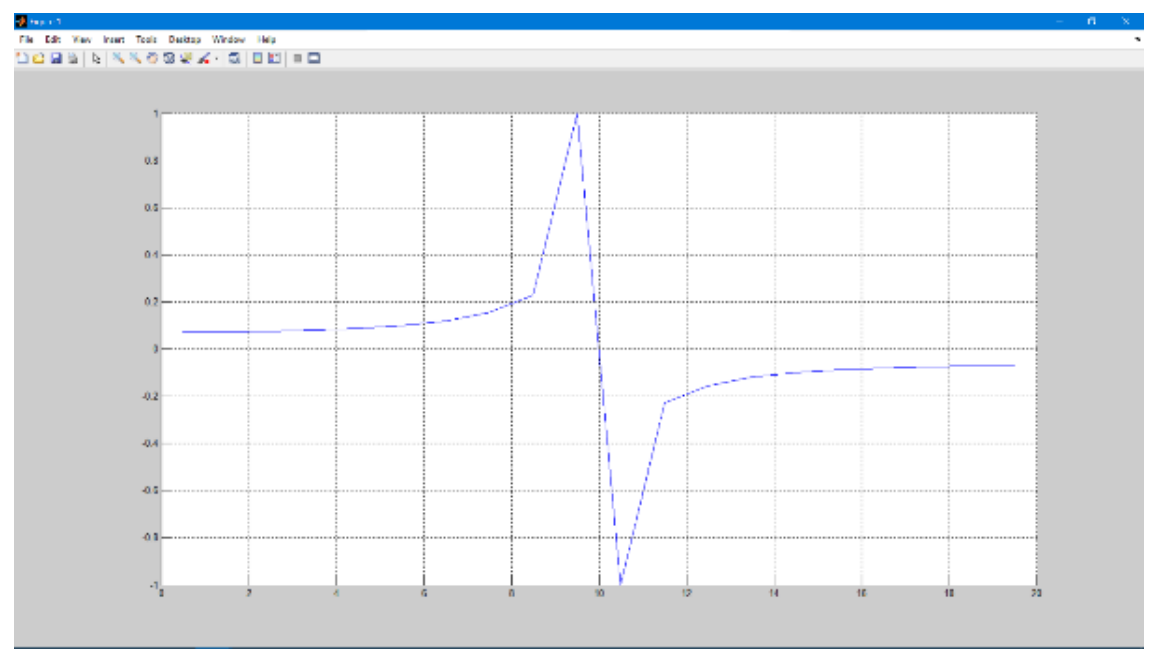

Figure 8. Surface Potential

\section{Conclusion}

The educational tool DITPRO has been created to simulate problems of direct problems in soil tomography, providing to the users an easy handling and powerful calculations. Its graphical environment allows access to different kind of graphical representation of potential. This type of learning tools designed for a specific problem facilitates the student's understanding of the problem. The student must to design and implement several real sceneries and explain it to check his/her understanding of the problem. 


\section{References}

Chapman, A. J. (1960) Heat transfer. New York: Macmillan Publishing Company.

González-Fernández C.F. and Horno J. (2002) Heat Transfer and the Network Simulation Method. Ed. Research Signpost, Kerala.

Mills, A. F. (1999) Heat transfer. New Jersey: Prentice Hall.

Sanchez-Perez, J.F., Alhama, I and Ramírez Medrano, F.A. (2016a) DITPRO (Direct Tomography program). Murcia, Spain: Intellectual Property Registration.

Sanchez-Perez, J.F. and Alhama, I (2016b) Teaching and learning of fundamental concepts of ground water flow by a specific educational software. 2nd International Conference on Higher Education Advances, HEAd’16. Procedia - Social and Behavioral Sciences $228,285-292$.

Sanchez-Perez, J.F., Conesa, M. and Alhama, I (2016) Solving ordinary differential equations by electrical analogy: a multidisciplinary teaching tool. European Journal of Physics, 37, 12 